\title{
A proposal of a He based Drift Chamber as central tracker for the IDEA detector concept for a future $e^{+} e^{-}$collider
}

\author{
F. Cuna, ${ }^{a, b, *}$ G. Chiarello, ${ }^{c}$ A. Corvaglia, ${ }^{b}$ N. De Filippis, ${ }^{e, f}$ E. Gorini, ${ }^{a, b}$ \\ F. Grancagnolo, ${ }^{b}$ A. Miccoli, ${ }^{b}$ M. Panareo, ${ }^{a, b}$ M. Primavera, ${ }^{b}$ G. F. Tassielli ${ }^{d, e}$ and
}

A. Ventura ${ }^{a, b}$

${ }^{a}$ Dipartimento di Matematica e Fisica "Ennio De Giorgi" - Universitá del Salento, Via Arnesano, 73100 Lecce, Italy

${ }^{b}$ Istituto Nazionale di Fisica Nucleare Sezione di Lecce, Via Arnesano, 73100 Lecce, Italy

${ }^{c}$ Istituto Nazionale di Fisica Nucleare Sezione di Pisa, Largo B. Pontecorvo 3, 56127 Pisa, Italy

${ }^{d}$ Dipartimento Interateneo di Fisica "Michelangelo Merlin", Università degli Studi di Bari "Aldo Moro", Piazza

Umberto I, 1, 70125 Bari, Italy

${ }^{e}$ Istituto Nazionale di Fisica Nucleare Sezione di Bari, Via E. Orabona 4, 70125 Bari, Italy

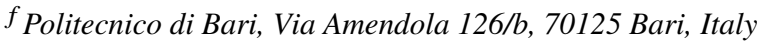

E-mail: federica.cuna@le.infn.it

The IDEA detector concept for a future $e^{+} e^{-}$collider adopts an ultra-low mass drift chamber as the central tracking system. It is a He based, $4 \mathrm{~m}$ long and $4 \mathrm{~m}$ diameter, fully stereo drift chamber with a total material budget of $\sim 0.016 X_{0}$ in the barrel part and $\sim 0.05 X_{0}$ in the end-caps. It will be instrumented with a readout electronics implementing the Cluster Counting/Timing techniques, allowing for a larger than $3 \sigma$ $\pi / \kappa$ separation over most of the momentum range of interest.

\footnotetext{
*** The European Physical Society Conference on High Energy Physics (EPS-HEP2021), ***

*** 26-30 July 2021 ***

*** Online conference, jointly organized by Universität Hamburg and the research center DESY ***
}

\footnotetext{
${ }^{*}$ Speaker
} 


\section{The IDEA detector and its innovative central drift chamber}

The IDEA detector (Innovative Detector for Electron-positron Accelerator) [1] is a general purpose detector which consists of a silicon pixel vertex detector, a large-volume extremely light drift chamber surrounded by a layer of silicon micro- strip detectors, a thin, low-mass superconducting solenoid coil, a $\mu$ Rwell pre-shower detector, a dual-readout calorimeter and a $\mu$ Rwell muon system inside the magnet return yoke. The IDEA drift chamber $(\mathrm{DCH})$ is designed to provide an efficient tracking, a high precision momentum measurement and an excellent particle identification by exploiting the application of the cluster counting technique. Main peculiarity of this drift chamber is its high transparency, in terms of radiation lengths, obtained thanks to the novel approach adopted for the wiring and assembly procedures. The total amount of material in radial direction, towards the barrel calorimeter, is of the order of $1.6 \% X_{0}$, whereas in the forward and backward directions it is equivalent to about $5 \% X_{0}$, including the endplates instrumented with front end electronics [2].

\subsection{The design and the novel approach for the construction technique}

The IDEA Central Drift Chamber (DCH) is a unique-volume, high granularity, fully stereo, low-mass cylindrical drift chamber, co-axial with the $2 \mathrm{~T}$ solenoid field, operating with an helium based gas mixture. It extends from an inner radius $R_{i n}=0.35 \mathrm{~m}$ to an outer radius $R_{\text {out }}=2 \mathrm{~m}$, for a length $L=4 \mathrm{~m}$ and consists of 112 co-axial layers, at alternating-sign stereo angles (see Figure 1), arranged in 24 identical azimuthal sectors, a layout similar to the one used in MEG II drift chamber [3].

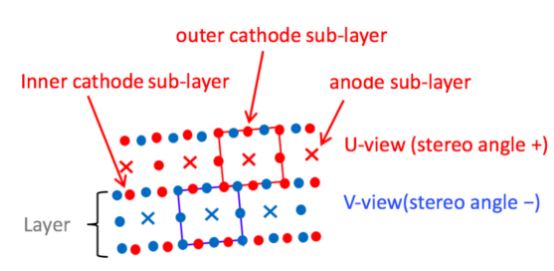

Figure 1: A sketch of the drift cells within two alternating sign stereo layers.

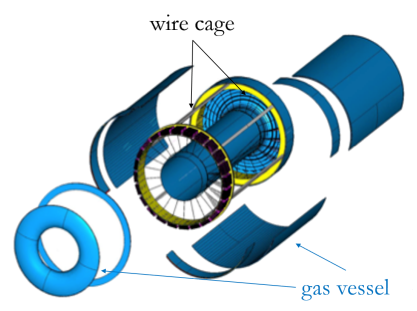

Figure 2: Schematic layout of the $\mathrm{CDCH}$ mechanical structure.

To accomodate the large number of wires, the design of wiring procedure and the new construction technique of MEG II [4] drift chamber will be used. To ensure the transparency, the gas containment function and the wire sustained function are separated: the wires are anchored to a self-sustained light structure (wire cage), which is surrounded by a thin skin of a suitable profile (gas vessel) to contain the gas mixture (see Figure 2). A system of tie-rods which directs the wire tension stress to the outer endplate rim, where a cylindrical carbon fiber support structure, bearing the total load, is attached.

\section{Cluster Counting/Timing and particle identification expected performance}

Counting the number of ionization acts per unit length $(\mathrm{dN} / \mathrm{dx})$ is a different method to identify the particles (P.Id.) with a better resolution than $\mathrm{dE} / \mathrm{dx}$ method. The analytic calculations in Figure 3 shows the particle separation power in terms of the numbers of standard deviation (sigma) as a function of momentum in a mixture of $90 \% \mathrm{He}$ and $10 \% i \mathrm{C}_{4} \mathrm{H}_{10}$. The solid curves refer to the separation with the cluster counting technique and the dashed curves refer to the optimal energy loss truncated mean technique. It is evident that a relative gain of a factor 2 of the cluster counting technique with respect to the $\mathrm{dE} / \mathrm{dx}$ method is reached. The study of the cluster counting techniques for helium based drift chambers requires the development of detailed simulations of the ionization process. Three different versions of an algorithm which, by using 


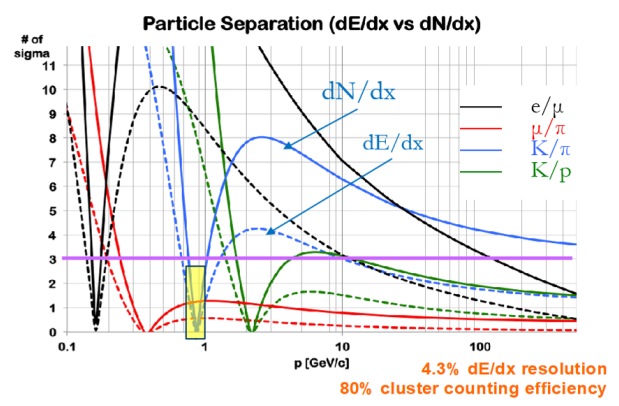

Figure 3: Analytic evaluation of particle separation capabilities achievable with $\mathrm{dE} / \mathrm{dx}$ (solid curves) and $\mathrm{dN} / \mathrm{dx}$ (dashed curves). The region between $0.85 \mathrm{GeV} / \mathrm{c}$ and $1.05 \mathrm{GeV} / \mathrm{c}$, where a different technique is needed, is highlighted in yellow.

the energy deposit information provided by Geant4, reproduce, in a fast and convenient way, the clusters number distribution and the cluster size distribution have been developed [5], thanks to detailed simulations of the ionization process with Garfield++ software tool for a gas mixture of $90 \% \mathrm{He}$ and $10 \% i C_{4} H_{10}$ [5]. Providing to the algorithms the energy loss simulated by Geant4,the number of clusters distribution and the cluster size distribution could be efficiently reconstructed. The simulations, in Geant4 and in Garfield++, confirm that the predictions about the cluster counting technique, as shown in Figure 4.
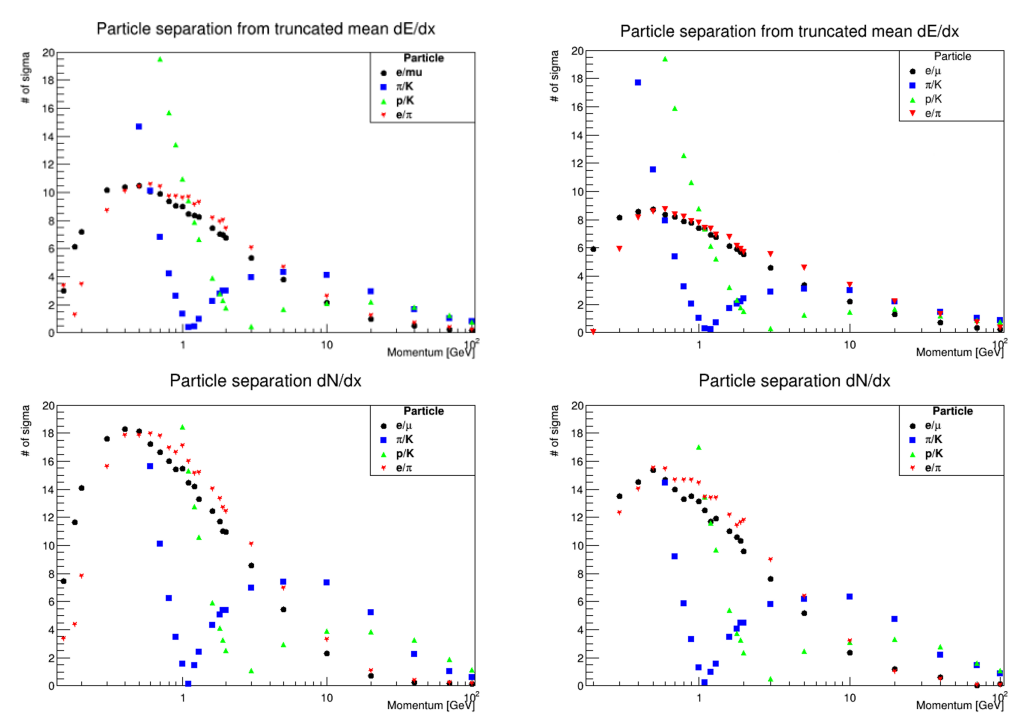

Figure 4: Top left: P.Id. obtained with the $\mathrm{dE} / \mathrm{dx}$ technique in Garfield++. Top right: P.Id. obtained with the $\mathrm{dE} / \mathrm{dx}$ technique in Geant4. Bottom left: P.Id. obtained with the $\mathrm{dN} / \mathrm{dx}$ method in Garfield++. Bottom right: P.Id. obtained with the $\mathrm{dN} / \mathrm{dx}$ method using one version of the algorithm implemented in Geant 4 .

\section{Tracking performance and fast simulations studies}

A Geant4 simulation has been performed to estimate the performance of the IDEA tracking system. Assuming a single cell resolution of $100 \mu \mathrm{m}$ for the chamber (as expected for the average single cell resolution [6]) and a conservative spatial resolution (pitch/ $\sqrt{12}$ ) for Si detectors, the IDEA tracking system meets the expected great performances, shown in Figure 5. The lightness of the drift chamber allows to the IDEA tracking system to gain almost a factor $\sim 3$ in momentum resolution respect to a full $\mathrm{Si}$ tracker system up to $\sim 50$ $\mathrm{GeV} / \mathrm{c}$, as shown in Figure 5 [2]. 

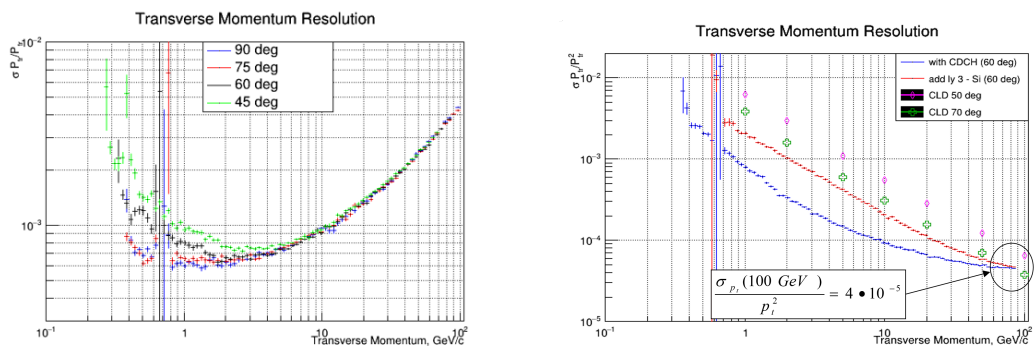

Figure 5: Left: Transverse momentum resolutions of the IDEA tracking system, evaluated for tracks with $\theta$ of $45^{\circ}, 60^{\circ}$ , $75^{\circ}$ and $90^{\circ}$. Right: Transverse momentum resolutions of the IDEA tracking system respect to a full Si based tracking system like the CLD detector one

A key measurement of the $e^{+} e-$ Higgs factories is the total cross section of $\mathrm{ZH}$ production. This is performed by measuring the $\mathrm{Z}$ momentum and by analyzing the recoil mass while using the collision energy as a constraint. In Figure 6 the recoil mass distribution expected with a perfect knowledge of the $\mathrm{Z}$ momentum is shown and compared to the expectations with the IDEA and CLD tracking systems [7], when the Z decays to two muons. The tracker performance in measuring the Higgs invariant mass in its two muon decay mode is shown in Figure 6 [8].
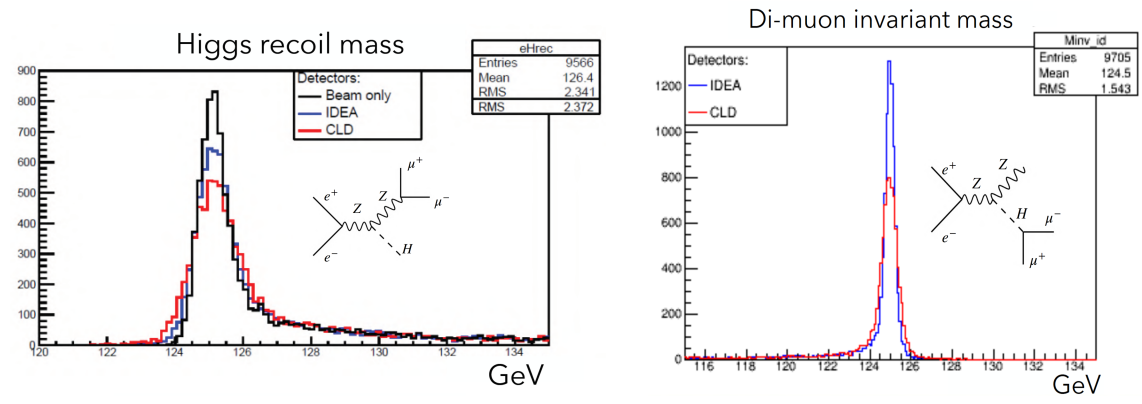

Figure 6: Left:Recoil mass distribution: (black) perfect detector, (blue) IDEA, (red) CLD. Right: Higgs invariant mass: (blue) IDEA, (red) CLD.

\section{References}

[1] M. Benedikt et al., FCC-ee The Lepton Collider : Future Circular Collider Conceptual Design-Report Volume 2. Eur. Phys. J. Spec. Top.228(2019) 261623.

[2] G.F. Tassielli on behalf of the IDEA Collaboration, A proposal of a drift chamber for the IDEA experiment for a future e+e- collider Vol. ICHEP2020, PoS. (2021)877.

[3] G. Chiarello on behalf of MEG collaboration, The construction technique of the high granularity and high transparency drift chamber MEG II, Journal of Instrumentation vol.12, C07022, July 2017.

[4] A. M. Baldini et al., The design of the MEG II experiment, Eur. Phys. J. C (2018) 78:380.

[5] F. Cuna et al., Simulation of particle identification with the cluster counting technique, in proceeding of International Workshop on Future Linear Collider arXiv:2105.07064 [physics.ins-det].

[6] A. M. Baldini et al., Single-hit resolution measurement with MEG II drift chamber prototypes, JINST 11 P07011 (2016).

[7] N. Bacchetta et al., CLD - A Detector Concept for the FCC-ee, arXiv:1911.12230 [physics.ins-det].

[8] F.Bedeschi, A detector concept proposal for a circular e+e-collider, Vol. ICHEP2020, PoS., 819. 